\title{
Reflets
}

Revue ontaroise d'intervention sociale et communautaire

\section{Brian L. Mishara et William A. McKim, Drogues et vieillissement, Boucherville, Gaëtan Morin éditeur, 1989}

\section{Michel-André Beauvolsk}

Volume 2, numéro 2, automne 1996

Vieillir à l'aube de l'an 2000

URI : https://id.erudit.org/iderudit/026144ar

DOI : https://doi.org/10.7202/026144ar

Aller au sommaire du numéro

Éditeur(s)

Reflets : Revue ontaroise d'intervention sociale et communautaire

ISSN

1203-4576 (imprimé)

1712-8498 (numérique)

Découvrir la revue

Citer ce compte rendu

Beauvolsk, M.-A. (1996). Compte rendu de [Brian L. Mishara et William A.

McKim, Drogues et vieillissement, Boucherville, Gaëtan Morin éditeur, 1989].

Reflets, 2(2), 190-192. https://doi.org/10.7202/026144ar

Tous droits réservés (C) Reflets : Revue ontaroise d'intervention sociale et communautaire, 1996
Ce document est protégé par la loi sur le droit d'auteur. L'utilisation des services d'Érudit (y compris la reproduction) est assujettie à sa politique d'utilisation que vous pouvez consulter en ligne.

https://apropos.erudit.org/fr/usagers/politique-dutilisation/ 


\section{Drogues et vieillissement}

\section{Brian L. Mishara et William A. McKim. Boucherville, Gaëtan Morin éditeur, 1989 \\ par}

\section{Michel-André Beauvolsk}

Université Laurentienne

Ce livre est une revue et une critique des recherches sur la consommation de médicaments et de drogues par les personnes âgées. Les auteurs tentent d'établir des liens entre les problématiques du vieillissement et la consommation de drogues prescrites ou non, des médicaments en vente libre et des drogues dites «récréatives». Il touche aussi les problèmes épidémiologiques, certains aspects psychologiques et sociologiques, les implications relatives à la prévention, en incluant une réflexion sur l'efficacité des drogues qui veulent préserver la jeunesse. Il présente aussi les programmes d'intervention et les changements dans les politiques sociales au cours des dernières années.

Le livre se compose de six chapitres. Le premier définit le concept clé, celui des drogues, la nomenclature des drogues et leurs effets de dépendance. Toute drogue devient une substance dont il sera question dans le livre. Leur appellation se fait en termes générique et commercial, et les auteurs définissent la dépendance physique et psychologique et le concept de vieillissement en termes d'âge chronologique, psychologique, émotif et d'âge social ${ }^{1}$.

Le deuxième chapitre propose une réflexion sur le vieillissement et la mort, et les efforts de la race humaine pour prévenir l'échéance de ces deux réalités de vie. Tout au long de l'histoire de l'humanité, la personne humaine a cherché des moyens de rester jeune : exercice physique, modifications du régime alimentaire, médecine et thérapies diverses. 
Le troisième chapitre discute des phénomènes de l'absorption, de la distribution, de l'élimination des drogues et de la sensibilité du corps humain à ces dernières. Les auteurs présentent au lecteur et à la lectrice une description des différents organes du corps humain qui participent à l'élimination des drogues.

Le quatrième chapitre fait un survol des drogues prescrites et des drogues en vente libre. Dans cette section du livre, les auteurs se penchent sur la fréquence de la consommation de drogues par les personnes âgées. Ils discutent de la relation entre médecins et patients, la prescription des médicaments et l'usage des médicaments par les personnes âgées. La relation, semble-t-il, n'est pas de dialogue mais plutôt un monologue dans lequel la personne âgée a peu de temps pour poser des questions, que ce soit sur les effets secondaires des médicaments ou sur leur usage prolongé.

Le cinquième chapitre couvre les drogues récréatives. Selon les auteurs, les personnes âgées semblent moins susceptibles de consommer de drogues récréatives que la génération des plus jeunes. Celles qu'elles utilisent sont légales: tabac, alcool et caféine en constituent l'essentiel.

Le sixième chapitre du livre propose un modèle de prévention et d'intervention des problèmes de drogue. Ici, les auteurs présentent la typologie classique de prévention primaire : éviter un problème avant qu'il ne se développe. L'intervention secondaire porte sur la réduction de l'importance des problèmes de drogue, et l'intervention tertiaire reflète les efforts menés par certains intervenants pour réduire l'impact d'un problème de drogue sur la vie d'une personne et d'une société. Mishara et McKim concluent que l'interaction indésirable de diverses médications, y compris l'alcool, constitue l'un des problèmes les plus graves causé par les médicaments. La recherche a montré que les personnes âgées reçoivent rarement l'information adéquate sur cette interaction ou sur la façon de reconnaître les problèmes reliés aux médicaments. Ainsi, non seulement faut-il informer les personnes âgées, on doit aussi s'assurer que les personnes âgées, aux prises avec un problème de santé dû à leur médication, reçoivent toute l'aide nécessaire. 
Le chapitre sept couvre les réflexions des auteurs sur les orientations de la pratique, des politiques et de la recherche sur les drogues et les personnes âgées. Les auteurs affirment que l'âge est important dans l'étude des effets des drogues et des médicaments lors du vieillissement. Tous ne vivent pas les mêmes effets au même rythme. Le défi des recherches futures réside justement dans la définition des différences inhérentes au processus de vieillissement et dans les réactions de chacun à l'usage des médicaments.

Les auteurs proposent plusieurs moyens de favoriser une consommation adéquate de médicaments et de drogues. Il reste à espérer qu'un jour, les organismes et services intéressés examineront de plus près la façon de prévenir les problèmes liés aux médicaments et autres drogues, car les personnes âgées ne reçoivent pas toujours d'aide adéquate.

Les auteurs terminent en rappelant qu'il existe de profondes différences individuelles dans tous les aspects des effets produits par les drogues et les médicaments, ainsi que dans la consommation par les personnes âgées. Ces constats sont pour eux les défis de la recherche dans l'avenir.

Les recherches présentées dans le document datent de quelques années. Nous aurions souhaité que des études plus récentes soient présentées. De plus, la traduction française laisse parfois à désirer. Toutefois, l'effort louable des auteurs de vouloir présenter un texte d'introduction sur la problématique des drogues et du vieillissement est intéressant. Un livre à lire, si l'on s'intéresse à un niveau élémentaire sur ce problème.

\section{Note}

1. Le vieillissement est démystifié, sans que les auteurs ne le définissent vraiment. 